Adandé Belarmain FANDOHAN ${ }^{1,2,3}$

Flora Josiane CHADARE ${ }^{4}$

Gerard Nounagnon GouWAKIN NOU ${ }^{5}$

Chénangnon Frédéric TovisSODE ${ }^{3}$

Alice BONOU 2,6

Spero Fréjus B. DJONLONKOU ${ }^{2}$

Loetitia F. H. HOUNDELO

Corine Laurenda B. SINSIN ${ }^{2}$

Achille Ephrem AssogBadjo ${ }^{2,3}$

1 Université nationale d'agriculture

École de foresterie et ingénierie du bois

Unité de recherche en foresterie,

agroforesterie et biogéographie

BP 43, Kétou

Bénin

2 Université d'Abomey-Calavi Faculté des sciences agronomiques Laboratoire d'écologie appliquée 05 BP 1752, Cotonou

Bénin

${ }^{3}$ Université d'Abomey-Calavi Faculté des sciences agronomiques Laboratoire de biomathématiques et d'estimations forestières 04 BP 1525, Cotonou Bénin

${ }^{4}$ Université nationale d'agriculture École des sciences et techniques de conservation et de transformation des produits agricoles BP 114, Sakété

Bénin

${ }^{5}$ Université de Parakou Faculté d'agronomie Laboratoire de recherche en écologie, botanique et biologie végétale BP 123, Parakou

Bénin

${ }^{6}$ African School of Economics (ASE) 02 BP 372, Cotonou

Bénin

\title{
Usages traditionnels et valeur économique de Synsepalum dulcificum au Sud-Bénin
}

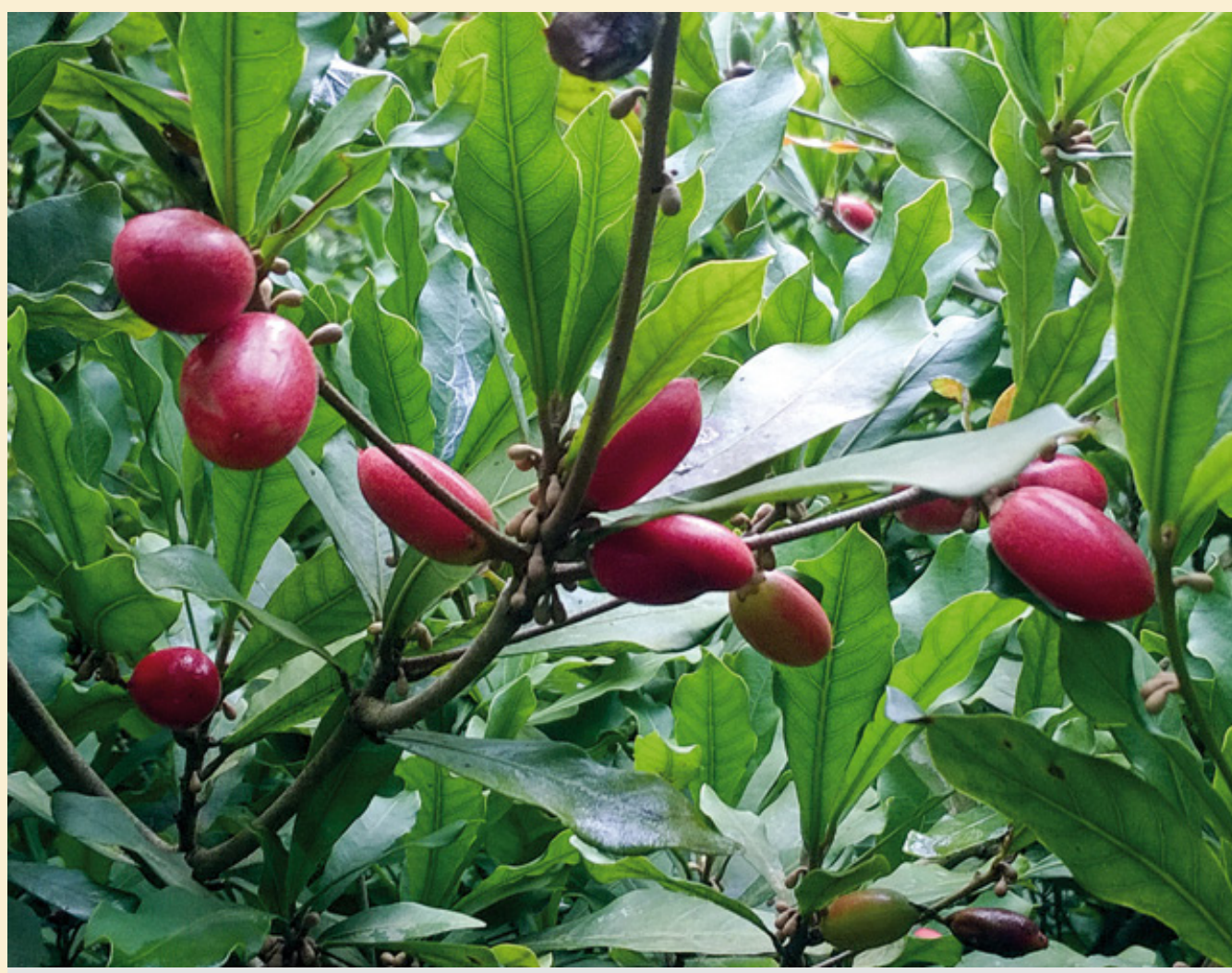

Photo 1.

Branche de Synsepalum dulcificum portant des fruits mûrs. Photo D. A. Tchokponhoué. 


\section{RÉSUMÉ}

\section{USAGES TRADITIONNELS ET VALEUR ÉCONOMIQUE DE SYNSEPALUM DULCIFICUM AU SUD-BÉNIN}

Synsepalum dulcificum (Schumach. \& Thonn. Daniell) est un arbuste originaire de l'Afrique de l'Ouest, inscrit sur la liste des espèces vulnérables de l'UICN. Au Bénin, son importance pour les populations locales reste peu documentée. L'étude avait pour objectif d'évaluer les connaissances endogènes, la valeur d'usage et l'importance économique de l'espèce pour les populations locales. Des enquêtes ethnobotaniques et économiques ont été conduites auprès de 606 personnes réparties dans 13 groupes socioculturels du Sud-Bénin. Des paramètres ethnobotaniques (fréquence de citation, valeur d'usage ethnobotanique) et économique (revenu moyen réalisé) ont été calculés, et leur significativité éprouvée par l'ajustement de modèles linéaires généralisés et le test de Kruskal et Wallis. Les résultats ont montré que $S$. dulcificum était bien connu des populations locales du Sud-Bénin (100 \% des enquêtés), qui le cultivaient notamment dans les jardins de case. Toutes les parties de la plante étaient utilisées à des fins médicinales, alimentaires et spirituelles. Les connaissances et la valeur d'usage de la plante variaient entre les groupes socioculturels du Sud-Bénin, avec un gradient décroissant Est-Ouest. Les connaissances et la valeur d'usage variaient suivant le sexe, l'âge et le domaine d'activité, les connaissances étant concentrées au niveau des hommes, des adultes et personnes âgées, et des praticiens de la médecine traditionnelle. L'évaluation économique a révélé un circuit de commercialisation relativement court. Le faible revenu moyen réalisé sur la vente des fruits (environ 28 USD par an et par commerçant) illustre la faible valeur économique de l'espèce qui constitue une ressource de subsistance en déclin. La conservation et la valorisation optimale de l'espèce nécessiteront des investigations sur les plans nutritionnel, phytochimique et pharmaceutique, phénologique, morphologique et génétique, le développement d'une sylviculture, l'intégration de la plante dans les politiques formelles de conservation, et enfin le développement d'une chaîne de valeurs à travers la mise en place d'une véritable filière.

Mots-clés : Synsepalum dulcificum, baie miraculeuse, enquête ethnobotanique, groupe socioculturel, phytothérapie, valeur d'usage, République du Bénin.

\section{ABSTRACT}

\section{TRADITIONAL USES AND ECONOMIC VALUE OF SYNSEPALUM DULCIFICUM IN SOUTH BENIN}

Synsepalum dulcificum (Schumach. \& Thonn. Daniell) is a West African shrub which is listed as vulnerable by the IUCN. Its importance for local people in Benin has been little documented. This study takes up this issue and was carried out to assess local knowledge, use value and the economic importance of the species for local people. Ethnobotanical and economic surveys were conducted with 606 respondents from 13 socio-cultural groups in southern Benin. Ethnobotanical and economic indices (citation frequency, ethnobotanical use value and mean income generated) were computed and their significance tested using generalized linear models and Kruskal and Wallis tests. The results showed that $S$. dulcificum was well known to local people in southern Benin (100\% of respondents), who mostly grew it in their home gardens. All parts of the plant were used, mostly for medicinal, food and spiritual purposes. Knowledge of the shrub and its use value varied among the socio-cultural groups, decreasing along a gradient from south-east to south-west. Knowledge and use value were also dependent on gender, age and activity, and concentrated among men, adults, elderly people and traditional healers. Economic data showed a short marketing chain. The low average income generated by selling the fruit (about US\$ 28 yearly per seller) reveals the low economic value of the species, which is a declining subsistence resource. Optimising the conservation and uses of the species would require (i) nutritional, phyto-chemical, pharmaceutical, phenological, morphological and genetic investigations, (ii) the development of sylvicultural method, (iii) inclusion of the species in formal conservation policies and (iv) development of a value chain by establishing a structured production channel.

Keywords: Synsepalum dulcificum, miracle fruit, ethnobotanical survey, socio-cultural group, phytotherapy, use value, Republic of Benin.

\section{RESUMEN}

\section{USOS TRADICIONALES Y VALOR ECONÓMICO DE SYNSEPALUM DULCIFICUM EN EL SUR DE BENÍN}

Synsepalum dulcificum (Schumach. \& Thonn. Daniell) es un arbusto originario de África Occidental clasificado como vulnerable en la lista de la UICN. Su importancia para la población local de Benín está poco documentada. El objetivo de este estudio consistía en evaluar el conocimiento local, su valor de uso y la importancia económica de la especie para la población local. Se efectuaron encuestas etnobotánicas y económicas a 606 personas de 13 grupos socioculturales del sur de Benín. Se calcularon los índices etnobotánicos (frecuencia de cita, valor de uso etnobotánico) y económico (ingreso promedio generado); y se probó su significación mediante el ajuste de modelos lineales generalizados y la prueba de Kruskal y Wallis. Los resultados evidenciaron que la población local del sur de Benín (100\% de encuestados) conocía bien $S$. dulcificum y la cultivaban sobre todo en huertos familiares. Se utilizan todas las partes de la planta para fines medicinales, alimentarios y espirituales. El conocimiento y valor de uso de la planta variaban entre los grupos socioculturales de la región, disminuyendo del Este al Oeste. El conocimiento y el valor de uso variaban también según el sexo, edad y tipo de actividad. Los hombres, adultos y ancianos, y los terapeutas tradicionales concentraban los mayores conocimientos. La evaluación económica reveló un circuito de comercialización relativamente corto. El bajo ingreso promedio que produce la venta de frutos (alrededor de 28 USD anuales por vendedor) muestra el bajo valor económico de la especie que constituye un recurso de subsistencia en declive. La conservación y valorización óptimas de la especie requieren: a) investigaciones nutricionales, fitoquímicas y farmacéuticas, fenológicas, morfológicas y genéticas; b) desarrollo de una silvicultura; c) integración de la planta en políticas formales de conservación y d) desarrollo de una cadena de valor mediante el establecimiento de un verdadero circuito de producción.

Palabras clave: Synsepalum dulcificum, baya milagrosa, encuesta etnobotánica, grupo sociocultural, fitoterapia, valor de uso, República de Benín. 


\section{Introduction}

La dépendance des populations locales africaines visà-vis des ressources prélevées en milieu naturel est avérée (Assogbadjo et al., 2008, 2009 ; Laleye et al., 2015), et peut représenter une menace pour la survie des espèces prélevées (Kalinganire et al., 2008 ; N'danikou et al., 2011). De nombreuses études se sont intéressées à la sauvegarde des moyens de subsistance des populations locales, se focalisant sur les aspects ethnobotaniques, écologiques et socio-économiques (Fandohan et al., 2011), mais aussi l'identification des espèces prioritaires à valoriser (N'danikou et al., 2011) ainsi que des espèces en danger (Adomou et al., 2011).

Synsepalum dulcificum (Schumach. \& Thonn.) Daniell est une sapotacée originaire d'Afrique de l'Ouest (Inglett et May, 1968), identifiée comme l'une des premières espèces fruitières locales susceptibles d'être domestiquées au Bénin (Adomou et al., 2011). Un intérêt croissant vise l'utilisation de son fruit, appelé « baie miraculeuse », dans la formulation d'aliments pour les diabétiques insulino-résistants, en raison de sa capacité unique à donner un goût sucré aux aliments acides sans apport équivalent d'énergie (Chen et al., 2006 ; Achigan-Dako et al., 2015 ; Shi et al., 2016).

La « baie miraculeuse » présente aussi un intérêt certain pour les industries cosmétiques et pharmaceutiques (Achigan-Dako et al., 2015). L'extrait des racines de la plante contient de l'acide vanillique (Chen etal., 2010) qui inhibe les cellules du cancer de la peau et présente une activité anti-oxydante à forte dose (Masson, 2014). La tige contient entre autres du ß-sitostérol et du stigmastérol qui présentent un effet anti-inflammatoire, antinéoplasique, antipyrétique et imuno-modulant chez les animaux (Sharma et al., 2014). À l'opposé des édulcorants artificiels tels que les cyclamates et la saccharine, la miraculine prévient les risques de carcinogenèse (IARC, 1980). Une synthèse des connaissances actuelles sur l'écologie, la propagation, les constituants chimiques de diverses parties de la plante, ainsi que les usages médicinaux avérés et potentiels de cette même espèce, a été dressée par Achigan-Dako et al. (2015).

Au Bénin, des études ont révélé l’importance de S. dulcificum, essentiellement aux plans médicinal et magique (Oumorou et al., 2010 ; Achigan-Dako et al., 2015). Cependant, l'espèce est actuellement inscrite sur la liste des espèces vulnérables de l'UICN (sous le nom Synsepalum glycydora), et les connaissances de base pour son utilisation durable demeurent encore limitées (Achigan-Dako et al., 2015). En effet, les informations disponibles sur l'écologie, la chorologie, la biologie et les formes d'usages traditionnels de la plante restent pour la plupart qualitatives (Akoègninou et al., 2006 ; Oumorou et al., 2010 ; Adomou et al., 2011). Les données relatives aux variations morphologiques et génotypiques intraspécifiques sont nécessaires à la conservation et la sélection des meilleurs cultivars (Achigan-Dako et al., 2015). De même, des informations quantitatives relatives aux utilisations des diverses parties de la plante, à la valeur économique locale, aux pressions et aux modes de gestion dans les systèmes agroforestiers traditionnels sont cruciales pour l'orientation de la gestion en faveur d'une utilisation durable (De Albuquerque et al., 2009). De plus, les informations sur la manière dont les caractéristiques sociales déterminent éventuellement l'exploitation d'une ressource apparaissent capitales pour apprécier l'importance de cette ressource pour les populations locales, et envisager la mise en place d'une filière de gestion (Honfo et al., 2015).

À ce titre, la présente étude visait à renforcer les connaissances sur S. dulcificum au Bénin. De façon plus spécifique, il s'agissait d'évaluer, d'une part, la valeur d'usage de $S$. dulcificum suivant le groupe socioculturel, le sexe, l'âge et le domaine d'activité, et, d'autre part, l'importance économique de l'espèce pour les populations locales du Sud-Bénin.

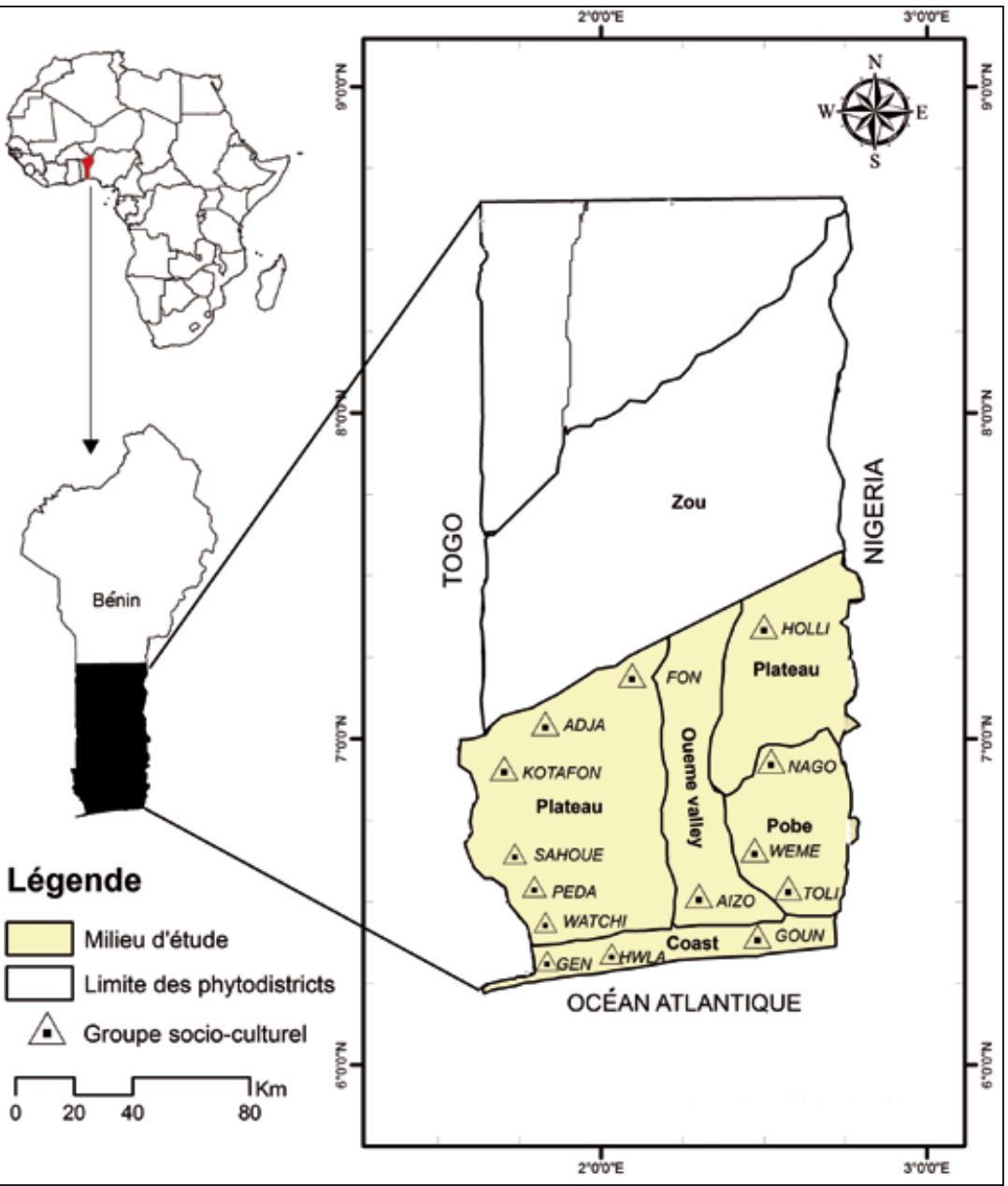

Figure 1.

Carte de situation de la zone d'étude. 


\section{Méthodes}

\section{Milieu d'étude}

La présente étude a eu pour cadre toute l'aire de distribution de $S$. dulcificum au Bénin (figure 1). Il s'agit des quatre districts phytogéographiques de la zone guinéo-congolaise au Bénin, compris entre $6^{\circ} 25$ et $7^{\circ} 30$ de latitude Nord (Akoègninou et al., 2006 ; Adomou et al., 2011). Le climat y est subéquatorial, avec deux saisons pluvieuses et deux saisons sèches. La pluviométrie annuelle varie de 900 mm à l'Ouest à 1300 mm à l'Est, et les sols dominants sont de type ferralitique et profond (Adomou, 2005).

S. dulcificum est rare à l'état sauvage au Bénin. L'espèce est surtout observée en zones cultivées près des habitations (Akoègninou et al., 2006). Les groupes socioculturels dominants dans le milieu d'étude sont les Adja, les Aïzo, les Fon, les Gèn, les Goun, les Holli, les Hwla (Péda compris), les Kotafon, les Nago (Yoruba compris), les Sahouè, les Toli, les Watchi et les Wémè. Ces populations vivent principalement de céréales (maïs, niébé), de tubercules (manioc, ignames), d'agrumes (oranges, citrons), de cultures maraîchères (tomate, piment) et de cultures de rente (coton, arachide, anacarde).

\section{Enquêtes ethnobotanique et économique}

Au total, 606 personnes ont été soumises à des entretiens individuels semi-structurés. Le nombre de personnes enquêtées a été déterminé par district phytogéographique. Pour ce faire, une enquête exploratoire a été réalisée auprès de 50 personnes choisies de façon aléatoire dans chacun des quatre districts phytogéographiques afin de déterminer la proportion $p$ de répondants connaissant $S$. dulcificum (figure 1). Le nombre $n$ d'enquêtés par district phytogéographique a été ensuite calculé comme suit (Dagnelie, 1998) :

$\mathrm{n}=\frac{\mathrm{U}_{0,975}^{2} \times \mathrm{p}(1-\mathrm{p})}{\mathrm{d}^{2}}($ Équation 1)

où $n$ est le nombre d'enquêtés pour un district phytogéographique donné ; $p$ est la proportion d'individus du district phytogéographique connaissant $S$. dulcificum $; U_{0,975} \approx 1,96$ est le quantile d'une distribution normale standard pour une valeur de probabilité de 0,05 ; et $d$ est l'erreur marginale, fixée à $8 \%$.

Le nombre de personnes enquêtées était de 171 dans le district du Plateau (29\%), de 164 dans le district côtier (27 $\%)$, de 136 dans le district de Pobè ( $22 \%$ ) et de 135 dans le district de la vallée de l'Ouémé (22\%). Les enquêtés ont été identifiés et leur permission a été obtenue avec l'aide des autorités locales. Les personnes propriétaires d'individus de $S$. dulcificum ont été systématiquement incluses dans l'échantillon enquêté. Des prospections ont été également conduites sur les marchés locaux afin d'identifier les commerçants de diverses parties de $S$. dulcificum.

Avec l'assistance d'un traducteur local au besoin, chaque enquêté a été soumis à un entretien semi-structuré à son domicile ou au marché, où les commerçants étaient plus souvent disponibles. Les données collectées par enquêté portaient sur ses caractéristiques sociales (groupe socioculturel, sexe, âge, occupation principale), la connaissance et la possession de $S$. dulcificum, les noms locaux attribués à la plante et leur signification, le mode d'acquisition, les habitats de l'arbuste en question et sa dynamique temporelle, les modes de propagation, les mesures de protection, les utilisations (parties de la plante concernées, formes d'usage) et l'importance relative des catégories d'usages.

L'importance des catégories d'usages a été évaluée à l'aide d'une grille affectant le score 1 pour les types d'usages peu ou pas pratiqués, le score 2 pour les types d'usages moyennement pratiqués, et le score 3 pour ceux les plus pratiqués. De même, cinq catégories d'usages ont été prédéfinies pour l'enquête : usage alimentaire, usage médicinal, utilisation comme brosse végétale, usage agricole et usage dans la magie (Oumorou et al., 2010).

Les 57 enquêtés commercialisant des parties de $S$. dulcificum ont été soumis à une enquête économique. À défaut de données sur toute une année, les données économiques fournies de mémoire incluent la saisonnalité de l'activité, les parties de plante vendues, les modes d'acquisition, les contraintes à l'activité, les minima et maxima des frais de transport, des quantités vendues, des prix de vente et du revenu généré.

\section{Traitement et analyses statistiques}

Les groupes socioculturels les plus représentés étaient les Fon, les Goun, les Nago, les Toli et les Adja. Les Fon ont été distingués en deux sous-groupes selon leur localisation, les «Fon1 » résidant dans le sud-est du Bénin, et les « Fon2 » résidant dans le sud-ouest. Plus de trois quarts des enquêtés (77\%) étaient des hommes, et l'âge médian des enquêtés était de 52 ans. Les personnes adultes (30 à 59 ans) et âgées (60 ans et plus) étaient les plus représentées (92\%) (tableau 1). Trois catégories socioprofessionnelles ont été distinguées : agriculteurs (58\%), commerçants (21\%), praticiens de la médecine traditionnelle (12\%). Une quatrième catégorie regroupe les autres activités ( $9 \%$, s'agissant de fonctionnaires et élèves) (tableau II). Les commerçants de parties de $S$. dulcificum étaient majoritairement des femmes (61\%), des adultes (37\%) et des jeunes (37\%).

Un modèle linéaire généralisé (GLM) basé sur la distribution binomiale a été ajusté à la possession d'individus de S. dulcificum (oui/non) afin de tester l'existence de relations significatives entre la possession et l'appartenance à un groupe social (groupe socioculturel, classe d'âge, sexe et domaine d'activité). Ensuite, un GLM basé sur la distribution binomiale négative a été ajusté au nombre d'individus par propriétaire, afin d'évaluer les variations du nombre d'individus de $S$. dulcificum par propriétaire entre et au sein des groupes socioculturels.

La fréquence de citation (FC) par l'ensemble des enquêtés a été utilisée pour évaluer l'importance relative de chaque utilisation de $S$. dulcificum. Seules les utilisations significatives $(F C \geq 0,05)$ ont été présentées en détail (Gouwakinnou et al., 2011). 
La valeur d'usage ethnobotanique (VUE ; Philips et Gentry, 1993) a été déterminée pour évaluer l'importance relative de chacune des catégories d'usages de $S$. dulcificum. La VUE $\mathrm{E}_{\mathrm{k}}$ représente la somme des scores d'usage $\mathrm{S}_{\mathrm{ik}}$ assignés par les enquêtés à une catégorie d'usages indexée $k$ divisée par le nombre total $\mathrm{N}$ d'enquêtés :

$\operatorname{VUE}_{\mathrm{K}}=\frac{\sum_{\mathrm{i}} \mathrm{S}_{\mathrm{ik}}}{\mathrm{N}}($ Équation 2)

L'intensité relative d'utilisation de $S$. dulcificum par un enquêté a été évaluée par la valeur d'usage ethnobotanique total (VUE ${ }_{T}$ ) qui est la somme des scores d'usage $S_{i k}$ assignés aux différentes catégories d'usages par l'enquêté :

$\mathrm{VUE}_{\mathrm{T}}=\sum_{\mathrm{k}} \mathrm{S}_{\mathrm{ik}}$ (Équation 3)
Les $\mathrm{VUE}_{\mathrm{T}}$ moyennes ont été calculées par groupe socioculturel, sexe, classe d'âge et domaine d'activité afin de décrire les variations de la valeur d'usage ethnobotanique de $S$. dulcificum entre et au sein des groupes socioculturels. Des tests de Kruskal et Wallis ont été réalisés sur la VUE afin de tester l'existence de différences significatives entre l'importance relative des catégories d'usages et entre l'importance relative de la plante, entre et au sein des groupes socioculturels.

Les commerçants de parties de $S$. dulcificum ont été distingués en deux groupes selon le type de donnée fournie : les détaillants (50\% des vendeurs) qui ont directement renseigné les revenus réalisés (minima et maxima) au cours d'une saison, et les semi-grossistes qui ont renseigné les prix et les quantités vendues (minima et maxima). Les détaillants

Tableau I.

Répartition des personnes enquêtées par groupe socioculturel, sexe et classe d'âge.

\begin{tabular}{|c|c|c|c|c|c|}
\hline $\begin{array}{l}\text { Groupe } \\
\text { socioculturel }\end{array}$ & \multicolumn{4}{|c|}{ Caractéristiques } & $\begin{array}{l}\text { Proportion de la population } \\
\text { totale enquêtée (\%) }\end{array}$ \\
\hline Goun & $\begin{array}{l}F \\
M\end{array}$ & $\begin{array}{l}2 \\
7\end{array}$ & $\begin{array}{l}11 \\
22\end{array}$ & $\begin{array}{l}12 \\
22\end{array}$ & 13 \\
\hline Nago & $\begin{array}{l}F \\
M\end{array}$ & - & $\begin{array}{r}9 \\
35\end{array}$ & $\begin{array}{r}3 \\
19\end{array}$ & 12 \\
\hline Fon2 & $\begin{array}{l}F \\
M\end{array}$ & $\begin{array}{l}4 \\
4\end{array}$ & $\begin{array}{r}9 \\
26\end{array}$ & $\begin{array}{r}6 \\
17\end{array}$ & 11 \\
\hline Toli & $\begin{array}{l}F \\
M\end{array}$ & $\begin{array}{l}1 \\
-\end{array}$ & $\begin{array}{r}8 \\
22\end{array}$ & $\begin{array}{r}8 \\
23\end{array}$ & 10 \\
\hline Fon1 & $\begin{array}{l}F \\
M\end{array}$ & $\begin{array}{l}2 \\
4\end{array}$ & $\begin{array}{r}6 \\
33\end{array}$ & $\begin{array}{r}4 \\
10\end{array}$ & 10 \\
\hline Adja & $\begin{array}{l}F \\
M\end{array}$ & - & $\begin{array}{r}7 \\
28\end{array}$ & $\begin{array}{r}3 \\
16\end{array}$ & 9 \\
\hline Kotafon & $\begin{array}{l}F \\
M\end{array}$ & $\begin{array}{l}1 \\
3\end{array}$ & $\begin{array}{r}4 \\
22\end{array}$ & $\begin{array}{r}4 \\
13\end{array}$ & 8 \\
\hline Aïzo & $\begin{array}{l}F \\
M\end{array}$ & - & $\begin{array}{r}3 \\
13\end{array}$ & $\begin{array}{r}1 \\
15\end{array}$ & 6 \\
\hline Wémè & $\begin{array}{l}F \\
M\end{array}$ & $\begin{array}{l}1 \\
4\end{array}$ & $\begin{array}{r}4 \\
12\end{array}$ & $\begin{array}{l}3 \\
8\end{array}$ & 5 \\
\hline Holli & $\begin{array}{l}F \\
M\end{array}$ & $\overline{1}$ & $\begin{array}{r}4 \\
14\end{array}$ & 9 & 5 \\
\hline Watchi & $\begin{array}{l}F \\
M\end{array}$ & - & $\begin{array}{r}5 \\
14\end{array}$ & $\begin{array}{l}2 \\
4\end{array}$ & 4 \\
\hline Hwla & $\begin{array}{l}F \\
M\end{array}$ & - & $\begin{array}{l}4 \\
7\end{array}$ & $\begin{array}{l}1 \\
3\end{array}$ & 3 \\
\hline Gen & $\begin{array}{l}F \\
M\end{array}$ & - & $\begin{array}{l}5 \\
4\end{array}$ & $\begin{array}{l}2 \\
3\end{array}$ & 2 \\
\hline Sahouè & $\begin{array}{l}F \\
M\end{array}$ & - & $\begin{array}{l}3 \\
7\end{array}$ & 4 & 2 \\
\hline $\begin{array}{l}\text { Proportion de la population } \\
\text { totale enquêtée (\%) }\end{array}$ & - & 8 & 56 & 36 & 100 \\
\hline
\end{tabular}


Tableau II.

Répartition des personnes enquêtées par domaine d'activité, sexe et classe d'âge.

\begin{tabular}{|c|c|c|c|c|c|}
\hline Domaine d'activités & Sexe & Jeune & Adulte & Vieux & $\begin{array}{c}\text { Proportion de la population } \\
\text { totale enquêtée (\%) }\end{array}$ \\
\hline Agriculture & $\begin{array}{l}F \\
M\end{array}$ & $\begin{array}{r}2 \\
33\end{array}$ & $\begin{array}{r}15 \\
193\end{array}$ & $\begin{array}{r}3 \\
105\end{array}$ & 58 \\
\hline Commerce & $\begin{array}{l}F \\
M\end{array}$ & $\begin{array}{l}7 \\
-\end{array}$ & $\begin{array}{l}60 \\
12\end{array}$ & $\begin{array}{r}40 \\
6\end{array}$ & 21 \\
\hline PMT & $\begin{array}{l}F \\
M\end{array}$ & - & 36 & 39 & 12 \\
\hline Autres activités & $\begin{array}{l}F \\
M\end{array}$ & $\begin{array}{l}2 \\
6\end{array}$ & $\begin{array}{r}7 \\
18\end{array}$ & $\begin{array}{r}6 \\
16\end{array}$ & 9 \\
\hline $\begin{array}{l}\text { Proportion de la population } \\
\text { totale enquêtée (\%) }\end{array}$ & - & 8 & 56 & 36 & 100 \\
\hline
\end{tabular}

$F=$ femme ; $M=$ homme ; Jeune (âge $<30$ ans) ; Adulte (30 $\leq$ âge $<60)$; Vieux (âge $\geq 60$ ans) ; $\mathrm{PMT}=$ praticiens de la médecine traditionnelle.

vendaient des tas de 5 à 40 unités de fruits, alors que les semi-grossistes vendaient les fruits par tas, mais aussi et surtout avec l'unité de mesure dénommée tooungolo (unité de volume souvent assimilée à environ un kilogramme), communément utilisée sur tous les marchés locaux du Bénin. La plupart des enquêtés n'ont pas renseigné les charges variables (frais de transport, coûts de la main-d'œuvre occasionnelle), ce qui a limité l'analyse économique à l'estimation des revenus moyens réalisés. La valeur économique (VE) d'une partie de la plante a été évaluée par le revenu saisonnier généré par cette partie (Bonou et al., 2013). Pour un détaillant et un semi-grossiste, les revenus par partie de la plante ont été calculés respectivement suivant les formules :

$\mathrm{R}_{\mathrm{i}}=\frac{\mathrm{R} \max _{\mathrm{i}}+\mathrm{R} \min _{\mathrm{i}}}{2}$ (Équation 4)

$R_{i}=\sum Q_{i} \times P_{i}($ Équation 5)

$\operatorname{Rmax}_{i}$ et Rmin ${ }_{i}$ étant respectivement les revenus maximal et minimal réalisés sur la vente de la partie indexée $i ; Q_{i}$ et $P_{i}$ étant respectivement les quantités moyennes vendues et les prix unitaires moyens de la partie indexée $i$ durant une saison donnée. $Q_{i}$ et $P_{i}$ ont été préalablement calculés en divisant les sommes des valeurs maximale et minimale par deux. La valeur économique globale de $S$. dulcificum a été déterminée comme la moyenne des revenus pour tous les commerçants de diverses parties de la plante.

Toutes les analyses statistiques ont été réalisées dans R-3.0.0 (R Core Team, 2014) et le seuil de significativité des tests a été fixé à $5 \%$. Les GLM ont été ajustés avec le package MASS (Venables et Ripley, 2002). Lors des ajustements, les interactions entre facteurs sociaux ont été éliminées au moyen de la fonction step de R afin de tester les hypothèses sur la base de modèles le plus parcimonieux possible. Les tests de Kruskal et Wallis ont été réalisés avec le package agricolae (De Mendiburu, 2014).

\section{Résultats}

\section{Appellations locales de $S$. dulcificum et signification}

Les noms locaux utilisés pour désigner $S$. dulcificum varient sans surprise selon les groupes socioculturels (tableau III), mais la signification reste la même et fait référence à la capacité du fruit à donner un goût sucré aux aliments amers ou aigres. À l'exception des groupes socioculturels Nago et Holli, les désignations de S. dulcificum étaient constituées de deux éléments distincts (séparés par un trait d'union dans le tableau III) : un radical qui est en fait le nom donné au fruit (photo 1) et un suffixe qui signifie arbre.

\section{Disponibilité, gestion et perceptions locales sur la dynamique de $\boldsymbol{S}$. dulcificum}

\section{Habitats de $S$. dulcificum}

Les habitats de $S$. dulcificum rapportés par les enquêtés étaient les jardins de case ( $83 \%$ des enquêtés), les champs et jachères sur terre ferme (36\%) ou en zones inondables (11\%). Au total, 373 individus de S. dulcificum ont été recensés, et la grande majorité (71\%) se trouvait dans les jardins de case (figure $2 a$ ). Le reste se situait près des abris de fortune construits au champ, en bordure de cours d'eau ou dans les bas-fonds.

\section{Mode d'acquisition et gestion de S. dulcificum dans les systèmes agroforestiers traditionnels}

La plupart des enquêtés (84\%) ont rapporté que $S$. dulcificum est une plante domestiquée. Les techniques de régénération citées sont le semis direct des graines (46\% des enquêtés) et le repiquage de jeunes plantules issues de la régénération naturelle (43\%). Les modes d'acquisition des individus de S. dulcificum étaient l'héritage (69\% des propriétaires), la plantation (31\%) et l'assistance à la 
Tableau III.

Appellations locales de Synsepalum dulcificum au sein des groupes socioculturels enquêtés.

\section{Groupes socioculturels}

\section{Appellations}

\begin{tabular}{lll|}
\hline Adja & Sinsi-tchi & Tanmini-tchi \\
\hline Kotafon & Sièsiè-tin & Atonmononmini-tin \\
\hline Gèn, Hwla et Peda & Sisrè-tin & \\
\hline Watchi & Sisrè-ti & Soussrouï-ti \\
\hline Fon1 et Fon2 & Sislè-tin & \\
\hline Goun & Assislè-tin & Ahihlè-tin \\
\hline Toli & Ahihlè-tin & \\
\hline Aïzo et Wémè & Sièsiè-tin & \\
\hline Holli et Nago & Agbayin & \\
\hline
\end{tabular}

régénération naturelle ( $4 \%$ ). L'héritage était cumulé avec la plantation chez $4 \%$ des propriétaires qui ont planté l'arbuste après en avoir hérité. Des plants de $S$. dulcificum en pépinière et des plantules de $S$. dulcificum sous semencier sont respectivement présentés sur les photos $2 \mathrm{a}$ et $2 \mathrm{~b}$.

Peu d'enquêtés (9\%) ont rapporté la mise en œuvre de mesures de protection contre des prélèvements illicites. Les diverses mesures individuelles citées concernent, d'une part, la protection de la plante par le sarclage régulier et la mise en place de pare-feu et d'enclos autour de l'arbuste, et, d'autre part, la mise en défens par l'accrochage à l'arbuste de talismans ou des emblèmes de fétiche Zangbéto. La mise en terre de l'arbuste à des endroits inaccessibles à tout le monde (arrière-cour et douche) permet également de le tenir hors des regards. Ces mesures visent à protéger la plante en évitant les prélèvements non autorisés par le propriétaire et destructeurs (feuilles et branches, écorce et racines) par les enfants, les praticiens de la médecine traditionnelle et tout autre utilisateur des diverses parties de la plante.

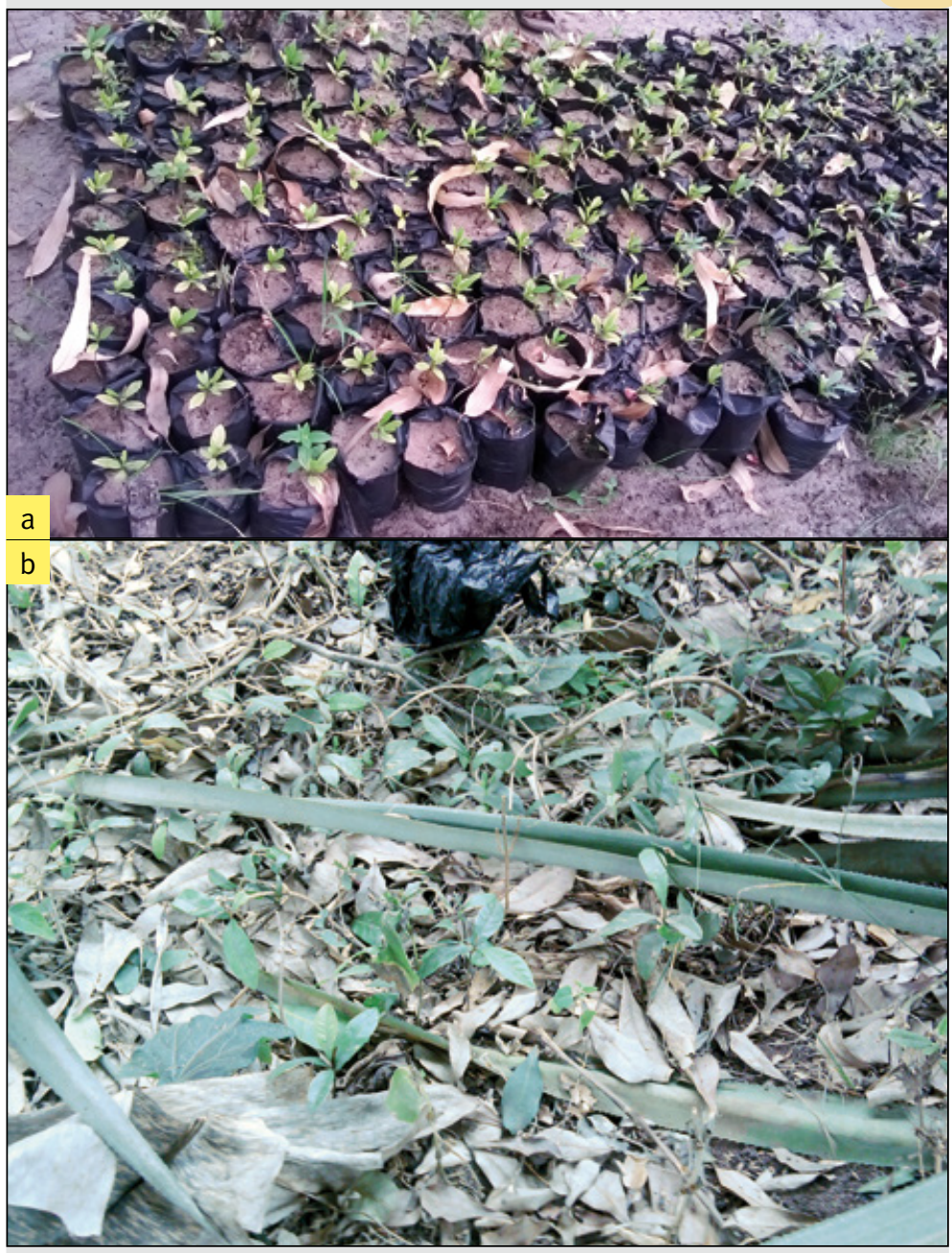

Photo 2.

Plants de Synsepalum dulcificum en pépinière (a) et plantules sous semencier (b).

Photo L. F. H. Houndelo.

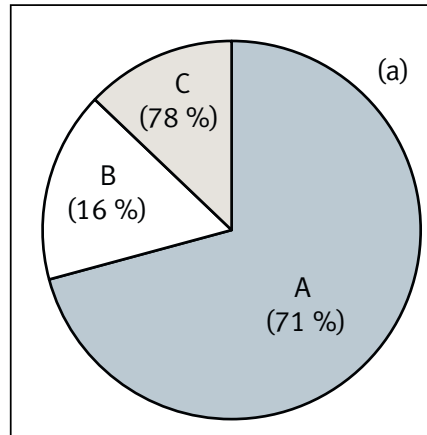

Habitats de Synsepalum dulcificum

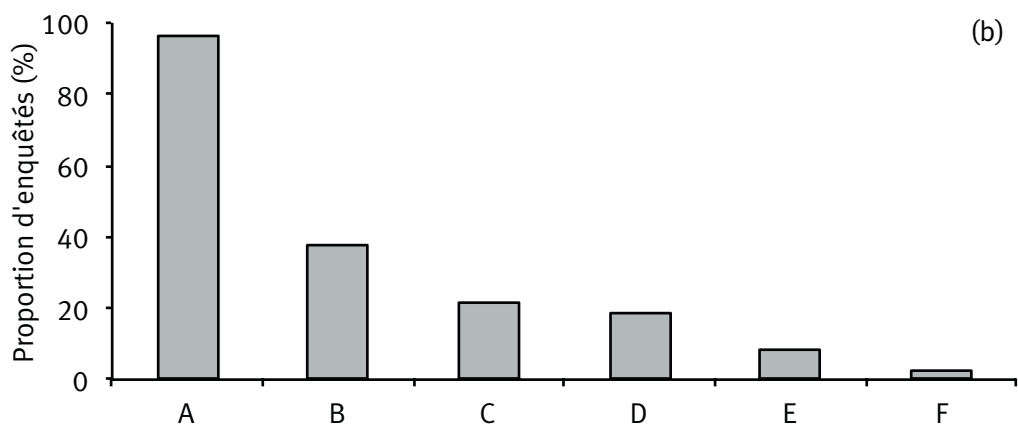

Causes du déclin de Synsepalum dulcificum
Figure 2.

Répartition suivant l'habitat des individus de Synsepalum dulcificum recensés (a) et facteurs responsables du déclin de $S$. dulcificum selon les enquêtés (b).

Partie (a) :

$A=$ jardin de case (y compris la cour, l'arrière-cour et la douche); $B=$ jachère ou champ (près des abris de fortunes construits pour le repos ou les séjours au champ durant les périodes de production); $C=$ champ ou jachère en zone inondable.

Partie (b) :

$A=$ faible importance économique; $B=$ ignorance des vertus de la plante; $C=$ récolte des parties de la plante; $D=$ tabous et représentations sociales; $E$ = pression démographique ; $\mathrm{F}=$ pression agricole et feux de végétation. 
Tableau IV.

Résultats de la régression binomiale sur la possession d’individus de Synsepalum dulcificum, des régressions binomiales négatives sur le nombre d'individus par propriétaire et des tests de Kruskal et Wallis sur la valeur d'usage ethnobotanique (VUE) de l'espèce au Sud-Bénin, selon le groupe socioculturel, le sexe, la classe d'âge et le domaine d'activité ( $\mathrm{DF}=$ degré de liberté ; $\mathrm{DFR}=$ degré de liberté résiduel ; Chi-2 = statistique Chi-2; $\mathrm{P}=$ probabilité).

\begin{tabular}{|c|c|c|c|c|}
\hline \multirow{3}{*}{$\begin{array}{l}\text { Caractéristiques } \\
\text { sociales des enquêtés }\end{array}$} & DF & DFR & Chi-2 & $\mathbf{P}$ \\
\hline & \multicolumn{4}{|c|}{ Possession d'individus de Synsepalum dulcificum } \\
\hline & 13 & 592 & 341,56 & $<0,001$ \\
\hline Sexe & 1 & 591 & 341,05 & 0,475 \\
\hline Classe d'âge & 2 & 589 & 333,84 & 0,027 \\
\hline \multirow[t]{2}{*}{ Domaine d'activité } & 3 & 586 & 332,71 & 0,771 \\
\hline & \multicolumn{4}{|c|}{ Nombre d'individus par propriétaire } \\
\hline Groupe socioculturel & 12 & 265 & 115,53 & 0,026 \\
\hline Sexe & 1 & 264 & 114,65 & 0,349 \\
\hline Classe d'âge & 2 & 262 & 112,77 & 0,389 \\
\hline \multirow[t]{2}{*}{ Domaine d'activité } & 3 & 259 & 112,26 & 0,916 \\
\hline & \multicolumn{4}{|c|}{ Valeur d'usage ethnobotanique (VUE) } \\
\hline Groupe socioculturel & 13 & & 289,76 & $<0,001$ \\
\hline Sexe & 1 & & 4,37 & 0,036 \\
\hline Classe d'âge & 2 & & 20,42 & $<0,001$ \\
\hline Domaine d'activité & 3 & & 21,44 & $<0,001$ \\
\hline
\end{tabular}

\section{Perceptions locales de la dynamique de $S$. dulcificum} dans les systèmes agroforestiers traditionels

La totalité des enquêtés (100\%) ont rapporté le déclin de la population de $S$. dulcificum. Les raisons justifiant ce déclin (figure 2 b) étaient surtout la faible importance économique de $S$. dulcificum (imputée à l'apparition du sucre à moindre coût sur le marché local par $17 \%$ des enquêtés) et l'ignorance des vertus de l'espèce. Environ un cinquième des enquêtés (21\%) attribuaient le déclin de la plante à des prélèvements destructeurs (racine et écorce). Les utilisations de $S$. dulcificum à des fins magiques génèrent des tabous, avec comme conséquence la crainte de planter, toucher ou utiliser la plante. La poussée démographique, la pression agricole et les feux de végétation constituaient les causes du déclin de $S$. dulcificum les moins citées.

\section{Variation de la possession d'individus de $S$. dulcificum entre et au sein des groupes socioculturels}

Au total, 278 enquêtés (46\%) possédaient au moins un individu de $S$. dulcificum. La fréquence des propriétaires variait significativement selon le groupe socioculturel et l'âge ( $P<0,05$; tableau IV). Par contre, la possession d'individus de S. dulcificum ne variait ni selon le sexe, ni selon le domaine d'activité des enquêtés ( $P>0,05$ ), et aucune interaction significative n'était notable entre les facteurs sociaux. Les figures $3 \mathrm{a}$ et $3 \mathrm{~b}$ présentent les proportions de propriétaires de $S$. dulcificum au sein des différents groupes socioculturels et des classes d'âge. Les propriétaires de $S$. dulcificum étaient plus fréquents (> $50 \%$ ) au sein des Adja, Hwla et Péda, Fon2, Kotafon, Sahouè et Watchi, avec des fréquences de propriétaires variant de $82 \%$ au sein des Adja à $68 \%$ au sein des Watchi. Au sein du groupe socioculturel Holli, aucun enquêté ne possédait d'individus de $S$. dulcificum. Les propriétaires étaient moins fréquents chez les jeunes (22\%) que chez les personnes de 30 ans et plus (48\%).

Le nombre d'individus de $S$. dulcificum par propriétaire variait de un à dix (médiane $=1$ ) et la plupart des propriétaires (84\%) possédaient un individu. Le nombre d'individus par propriétaire variait significativement selon le groupe socioculturel ( $P=0,026$; tableau IV), mais aucune variation, ni aucune interaction n'étaient notables au regard du sexe, de l'âge ou du domaine d'activité ( $P>0,05)$. La figure $3 c$ présente le nombre moyen d'individus de $S$. dulcificum par propriétaire au sein des différents groupes socioculturels. Les propriétaires Aïzo, Toli, Goun et Fon1 possédaient plus d'individus de $S$. dulcificum (médiane $=1$ à 2 individus) que les propriétaires des autres groupes socioculturels. Au sein du groupe socioculturel Hwla, tous les propriétaires possédaient chacun un individu de $S$. dulcificum.

\section{Connaissances et valeur d'usage de $S$. dulcificum}

\section{Diversité des utilisations et importance des parties et catégories d'usages de $S$. dulcificum}

Soixante-quatre utilisations de $S$. dulcificum ont été signalées lors des enquêtes ethnobotaniques. Mais seulement neuf utilisations avaient des fréquences de citation significatives ( $F C \geq 5 \%$; tableau V). L'utilisation alimentaire du fruit (édulcorant) était connue de tous les enquêtés (FC= $100 \%)$. L'utilisation médicinale des racines en macération 

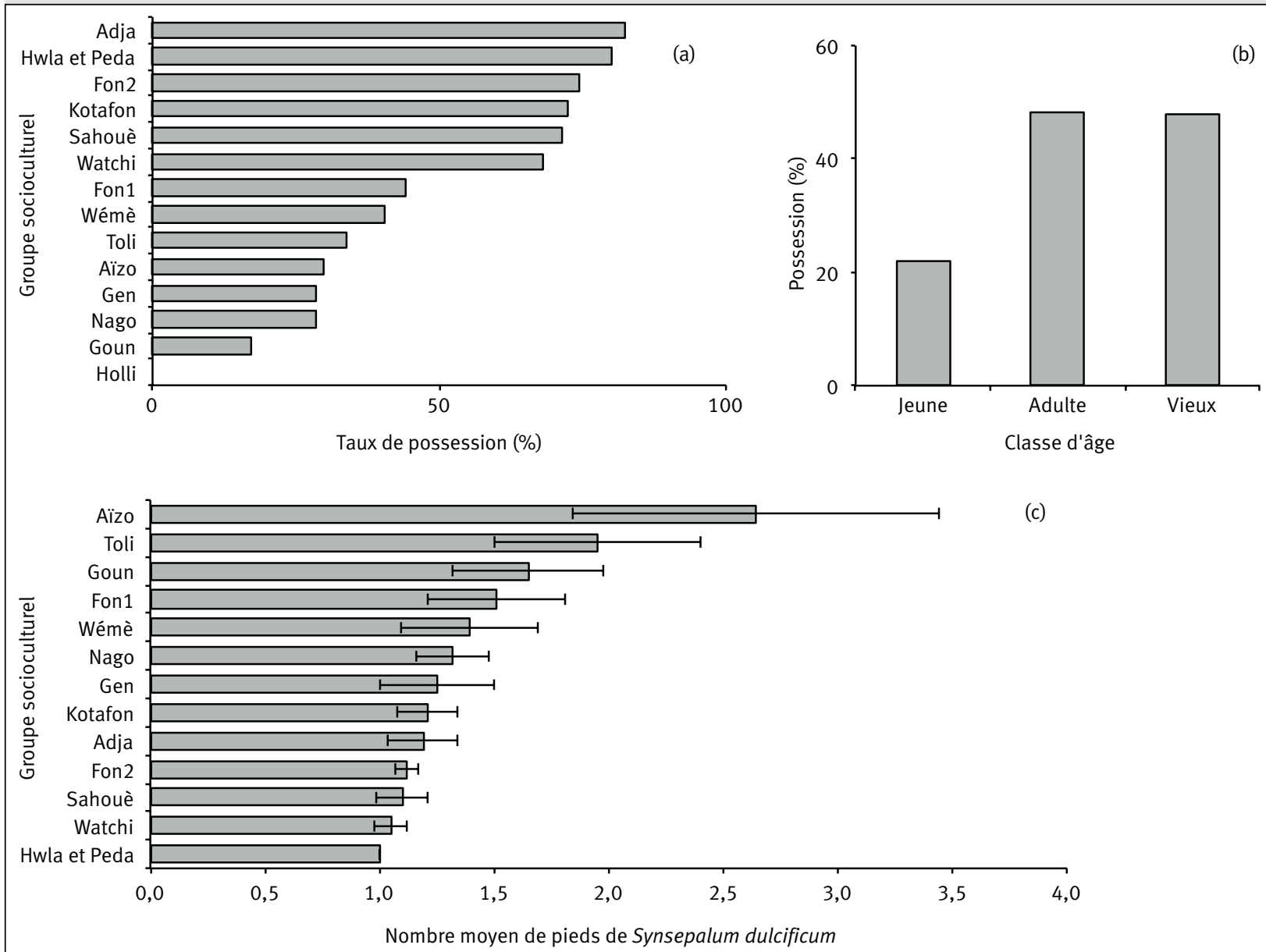

Figure 3.

Possession de Synsepalum dulcificum selon le groupe

socioculturel (a) et la classe d'âge (b) et nombre moyen

( \pm erreur type) des individus par propriétaire (c).

\section{Tableau V.}

VUE $_{k}$ (Valeur d'usage ethnobotanique) des catégories d'usages de Synsepalum dulcificum, utilisations significativement rapportées ( $F C \geq 5 \%$ ) et $F C$ (fréquence de citation), parties impliquées, préparations (+ indique l'addition d'ingrédients non précisés; macération sans autre précision indique une macération dans l'eau) et voie/forme d'usage au Sud-Bénin.

\begin{tabular}{|c|c|c|c|c|c|c|}
\hline Catégorie d'usage & VUE $_{k}$ & Partie & Utilisation / maladie soignée & Préparation & Voie / Forme & FC \\
\hline \multirow[t]{4}{*}{ Médicinale } & 1,38 & Racine & Faiblesse sexuelle & Macération (+) dans du vin de palme & Orale & 20 \\
\hline & & Feuille & Enurésie & Décoction (+) & Orale & 17 \\
\hline & & & Paludisme & Décoction (+) & Orale & 11 \\
\hline & & & Ictère & Décoction (+) & Orale & 7 \\
\hline Alimentaire & 1,19 & Fruit & Édulcorant & Consommer cru (avant aliment aigre/amer) & Orale & 100 \\
\hline \multirow[t]{3}{*}{ Magique } & 0,70 & Feuille & Attirer des clients & Mâché (+) & Orale & 13 \\
\hline & & Feuille & Fidéliser la femme & Trituration (+) & Massage & 5 \\
\hline & & Tige & Se faire une bonne réputation & Mâché (+) & Orale & 6 \\
\hline Brosse végétale & 0,20 & Tige & Brosse végétale & Brosse végétale & Orale & 12 \\
\hline
\end{tabular}


pour le traitement de la faiblesse sexuelle $(F C=20 \%)$ et des feuilles en décoction pour le traitement de l'énurésie (FC = $17 \%$ ) et du paludisme ( $\mathrm{FC}=11 \%$ ), l'utilisation magique des feuilles pour s'attirer la clientèle ( $F C=13 \%)$ et l'utilisation de la tige comme brosse à dents végétale ( $F C=12 \%)$ étaient les usages non alimentaires les plus répandus.

La diversité des utilisations de $S$. dulcificum variait suivant la partie de plante impliquée. Les parties participant aux usages significatifs étaient les feuilles, la tige, les fruits et les racines (respectivement cinq, deux, un et un usages significatifs). Les parties recherchées pour les différentes utilisations étaient principalement les feuilles, qui participaient à 45 usages sur les 64 répertoriés (soit $70 \%$ ). Les racines, la tige et l'écorce étaient respectivement impliquées dans 22, 10 et 10 usages. Les parties impliquées dans le moins d'usages étaient le fruit (six usages), la graine (cinq usages) et la fleur (un usage).

La diversité des utilisations de $S$. dulcificum variait également suivant la catégorie d'usages. Les usages significatifs ( $F C \geq 5 \%$ ) étaient surtout médicinaux (44\%) et magiques (33\%). Aussi, la majorité des 64 utilisations répertoriées étaient des usages médicinaux (70\%) et magiques (22\%). Les valeurs d'usage ethnobotanique (VUE $)$ déterminées pour les différentes catégories d'usages (tableau $V$ ) ont révélé que S. dulcificum était surtout sollicité pour des utilisations médicinales $\left(V^{\prime} E_{k}=1,38\right)$ et alimentaires $\left(\operatorname{VUE}_{k}=1,19\right)$. Ces deux groupes d'usages étaient significativement plus pratiqués (test de Kruskal et Wallis ; $D F=4$; Chi- $2=1308,42$; $P<0,001$ ) que les usages à des fins magiques $\left(\mathrm{VUE}_{k}=0,67\right)$. L'utilisation de la tige comme brosse à dents végétale $\left(\mathrm{VUE}_{\mathrm{k}}=0,19\right)$ et les utilisations agricoles $\left(\mathrm{VUE}_{\mathrm{k}}=0,08\right)$ étaient rarement pratiquées.

\section{Valeur d'usage ethnobotanique de S. dulcificum et variations entre et au sein des groupes socioculturels}

Les résultats des tests de Kruskal-Wallis sur la valeur d'usage ethnobotanique (VUE) de S. dulcificum (tableau IV) indiquent que l'intensité d'utilisation de la plante par les enquêtés variait significativement suivant le groupe socioculturel (DF $=13$; Chi-2 = 289,76; $\mathrm{P}<0,001)$, le sexe (DF = 1 ;
Chi-2 = 4,37; $P=0,036)$, l'âge ( $D F=2$; Chi-2 = 20,42 ; $P<0,001)$ et le domaine d'activité ( $D F=1$; Chi-2 = 21,44; $P<0,001)$ mais aucune interaction significative n'était notable entre ces facteurs sociaux.

Les VUE moyennes sont présentées aux figures 4a-d. Les groupes socioculturels utilisant le plus $S$. dulcificum étaient les Fon1, les Goun, les Nago, les Toli, les Holli, les Aïzo et les Wémè (VUE > 3). Ainsi, les hommes (VUE $=3,57$ ) utilisaient plus $S$. dulcificum que les femmes (VUE = 3,18); les personnes de 60 ans et plus (VUE $=3,93$ ) l'utilisaient plus que les adultes (VUE $=3,28$ ) et les jeunes (VUE $=2,90)$. De même, les praticiens de la médecine traditionnelle (VUE = 4,47 ) utilisaient $S$. dulcificum plus que les personnes relevant d'autres domaines d'activité (VUE <3,5).

\section{Parties commercialisées et valeur économique de $S$. dulcificum}

\section{Parties à valeur économique, modes d'acquisition et contraintes à la commercialisation}

Au Sud-Bénin, les parties de S. dulcificum commercialisées étaient le fruit (92\% des vendeurs), la feuille (6\%) et les jeunes plants produits par des pépiniéristes (3\%). Le principal mode d'acquisition de ces parties par les vendeurs était la cueillette (89 \% des vendeurs). L'achat (8\%) et le don (3\%) des diverses parties de S. dulcificum étaient peu fréquents. La clientèle comprenait des enfants friands des fruits (54\% des clients), des détaillants (27\%), des semi-grossistes constitués de vendeurs d'oranges (15\%) et des praticiens de la médecine traditionnelle (3\%). Les fruits étaient toujours vendus frais, alors que les feuilles étaient vendues fraîches ou sèches.

Le prix des tas de 5 à 40 unités de fruits variait de 0,01 à 0,05 USD. Quant au tooungolo de fruits, le prix unitaire variait de 0,25 USD en milieu rural à 6 USD en milieu urbain et périurbain. Les prix et l'unité de vente des feuilles ainsi que le prix de vente des plants de $S$. dulcificum en pépinière n’ont pas été renseignés par les vendeurs.
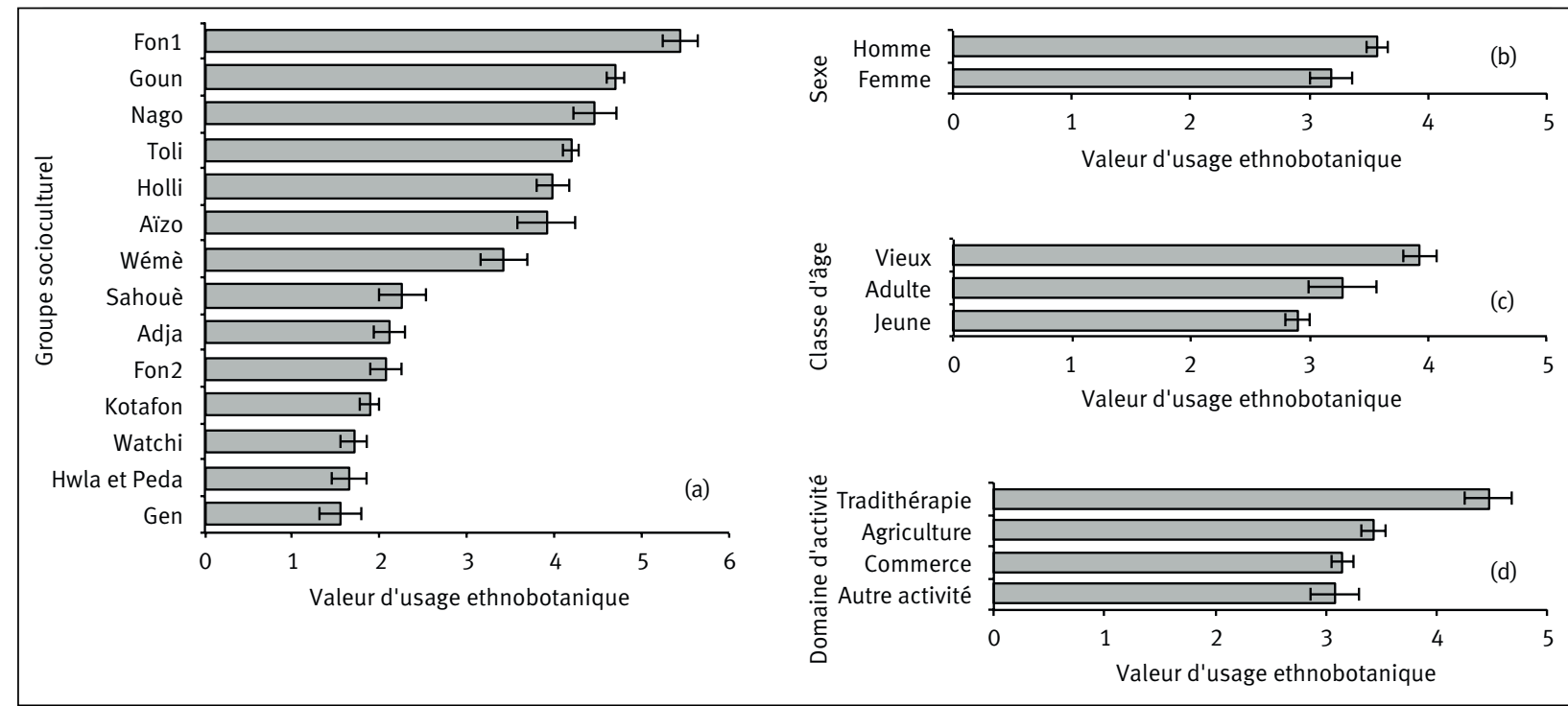

Figure 4.

Valeur d'usage ethnobotanique (VUE \pm erreur type) de Synsepalum dulcificum par groupe socioculturel (a), sexe (b), classe d'âge (c) et domaine d'activité (d). 
Les contraintes liées à la commercialisation des parties de $S$. dulcificum étaient le caractère très périssable du fruit (54\% des vendeurs) et la faiblesse de la demande (43\%).

\section{Valeur économique du fruit de $S$. dulcificum}

En raison du manque d'information sur les prix et les quantités de feuilles et plants en pépinière vendus, seul le fruit de $S$. dulcificum a été considéré pour l'évaluation de la valeur économique de S. dulcificum (tableau VI). Les commerçants de parties de $S$. dulcificum ont indiqué la disponibilité du fruit toute l'année, mais distinguaient deux saisons correspondant aux pics de production observés aux mois de juin et d'octobre. Le revenu saisonnier réalisé par un détaillant de fruits de $S$. dulcificum variait de 10 USD à 40 USD pour une moyenne de 16,97 USD. Quant au semi-grossiste, le volume de fruits vendu par saison variait de 2 à 80 tooungolo (environ 2 à 80 kilogrammes) et le revenu saisonnier moyen réalisé était de 8,70 USD. Globalement (détaillants et semi-grossistes réunis), la valeur économique de $S$. dulcificum a été estimée à 13,87 USD par saison, soit environ 27,74 USD par an.

\section{Discussion}

\section{Limites de l'étude}

Cette étude a exploité des données d'enquêtes conduites auprès des populations locales du Sud-Bénin afin d'établir si les connaissances et la valeur d'usage de $S$. dulcificum variaient entre et au sein des groupes socioculturels. Le ciblage systématique des personnes propriétaires d'individu(s) de $S$. dulcificum et des commerçants de parties de S. dulcificum constitue certes un bais dans les résultats de l'étude, en particulier les tests statistiques requérant l'indépendance des échantillons (Espinosa et al., 2012).

Cependant, les personnes systématiquement impliquées constituaient une source privilégiée d'informations sur la commercialisation des parties de la plante ainsi que sa disponibilité. Leur implication s'apparente donc à l'implication systématique des praticiens de la médecine traditionnelle dans de nombreuses études ethno-médicinales en raison de leur connaissance avérée des utilisations traditionnelles des plantes (Laleye et al., 2015).

\section{Les populations locales du Sud-Bénin sont conscientes du déclin de $S$. dulcificum}

S. dulcificum est principalement cultivée dans les jardins de case et les champs, ce qui corrobore les informations déjà disponibles sur l'habitat de l'espèce au Bénin (Akoègninou et al., 2006 ; Oumorou et al., 2010 ; Adomou et al., 2011 ; Achigan-Dako et al., 2015). Le mode d'acquisition dominant est l'héritage (69\%), mais près du tiers des individus recensés (31\%) ont été plantés par les propriétaires, ce qui traduit l'intégration de la culture de l'espèce dans les pratiques agroforestières (Achigan-Dako et al., 2015).

Les populations locales du Sud-Bénin sont conscientes (100 \% des enquêtés) du déclin de S. dulcificum signalé par Adomou et al. (2011). Mais, en dépit de leur perception du déclin de l'espèce, les acteurs impliqués dans l'utilisation intense des parties de la plante ne semblent pas craindre sa disparition. Les propriétaires, impuissants face aux prélèvements (feuilles et branches, écorce et racines) sans leur avis, ont recours aux pratiques cultuelles de dissuasion par accrochage de talismans et d'emblèmes de la divinité Zangbéto, reconnue comme gardien dans le Sud-Bénin. Mais ces moyens de dissuasion sont de moins en moins efficaces, conduisant à la plantation en arrière-cour et à proximité des douches, seul moyen de mettre la plante à l'abri de prélèvements illicites et destructeurs. Cette situation est particulièrement critique pour une espèce pratiquement absente à l'état naturel (Adomou et al., 2011), et dont la survie dépend exclusivement de son intégration et de son maintien dans les systèmes agroforestiers locaux.

En outre, $S$. dulcificum est surtout propagé au Bénin par semis direct (Achigan-Dako et al., 2015). Ses graines sont réputées récalcitrantes à la conservation (Ogunsola et Ilori, 2008). Ceci n'encourage pas les populations à planter l'espèce. La propagation par bouturage, greffage ou marcottage (Joyner, 2006) ainsi que la micropropagation (Ogunsola et Ilori, 2008) permettent une fructification plus rapide. La mise au point et la vulgarisation de telles techniques au Bénin pourraient stimuler la culture de l'espèce par les populations locales. Toutefois, la propagation par graines permet des recombinaisons génétiques (Jaenicke et Beniest, 2002) et reste ainsi indispensable pour la conservation de la diversité génétique de S. dulcificum (Achigan-Dako et al., 2015).

Tableau VI.

Quantité vendue par saison, prix de vente unitaire (PVU), revenu moyen (R) réalisé par catégorie de vendeurs par saison sur la vente de fruits, et valeur économique (VE) de Synsepalum dulcificum au Sud-Bénin.

\begin{tabular}{l|c|c|c|c|c|}
\hline $\begin{array}{l}\text { Catégorie } \\
\text { de vendeurs }\end{array}$ & $\begin{array}{l}\text { Quantité (tooungolo) } \\
\text { Min }\end{array}$ & \multicolumn{2}{c|}{ PVU (USD) } & R (USD) & VE (USD) \\
\hline Détaillant & - & Min & Max & \\
\hline Semi-grossiste & 2 & - & 0,01 & 0,05 & 16,97 \\
\hline
\end{tabular}




\section{Les utilisations majeures de $S$. dulcificum sont médicinales}

S. dulcificum est une espèce à usages multiples au Sud-Bénin, les domaines d'utilisation allant de la phytomédecine et l'alimentation humaine (Oumorou et al., 2010) à l'agriculture en passant par l'usage spirituel et l'hygiène bucco-dentaire. Les appellations locales de S. dulcificum spécifiques à des groupes socioculturels traduisent comment les populations locales perçoivent la plante et son potentiel, sur le plan alimentaire et dans la sphère spirituelle (NB : les utilisations magiques visant à " attirer des clients ", " fidéliser la femme » ou "se faire une bonne réputation » sont associées au goût sucré du fruit par les enquêtés).

La présente étude a révélé que, même si l'utilisation alimentaire de la baie miraculeuse comme édulcorant est l'usage le plus répandu, $S$. dulcificum est plus intensément utilisé à des fins médicinales qu'alimentaires. Les usages médicinaux les plus répandus sont le traitement de troubles du système urogénital (faiblesse sexuelle et énurésie) et des infections (paludisme, ictère). Ces usages médicinaux étaient jusqu'alors non documentés et pourraient être investigués par des études phytochimiques. L'utilisation potentielle du fruit dans l'élaboration d'aliments pour les diabétiques insulino-résistants (Chen et al., 2006) était inconnue des enquêtés, même si l'utilisation des feuilles de S. dulcificum dans le traitement du diabète a été mentionnée par quelques-uns (0,5\%).

\section{Les connaissances et la valeur d'usage de S. dulcificum dépendent du groupe socioculturel}

Les résultats des tests statistiques réalisés ont montré que les connaissances endogènes et la valeur d'usage de $S$. dulcificum varient entre les groupes socioculturels. Les groupes Fon1 (résidant dans le Sud-Est), Holli et Toli ont globalement plus de connaissances sur S. dulcificum (en moyenne trois à quatre usages par enquêté) que les autres groupes socioculturels du Sud-Bénin. Les Nago/Yoruba, les Wéme, les Goun et les Aïzo, dont les enquêtés résident majoritairement dans le Sud-Est du Bénin comme les Fon1, Holli et Toli, connaissent également plus d'usages de $S$. dulcificum que les autres groupes socioculturels dont les enquêtés résident majoritairement dans le Sud-Ouest du pays (Sahouè, Adja, Fon2, Kotafon, Watchi, Hwla/Péda et Gèn). De plus, les groupes socioculturels du Sud-Est présentent les plus fortes intensités d'utilisation de la plante. Ces résultats suggèrent que les différences interculturelles observées dans la connaissance des usages de $S$. dulcificum sont reliées à l'environnement et à la disponibilité de la ressource végétale (Avocèvou-Ayisso et al., 2011). Cette explication semble d'autant plus plausible qu'en dépit de leur appartenance à un même groupe socioculturel, les Fon1 résidant dans le SudEst connaissent plus d'usages et utilisent plus intensément les parties de $S$. dulcificum que les Fon 2 résidant dans le SudOuest. Plusieurs études sur la connaissance des usages des ressources végétales ont en effet rapporté des différences interculturelles attribuées à l'abondance de la ressource dans le milieu des groupes socioculturels les mieux informés (Gouwakinnou et al., 2011 ; Laleye et al., 2015).
Dans le cas d'étude, la possession de $S$. dulcificum est effectivement négativement corrélée avec l'utilisation de la plante, avec davantage de propriétaires au sein des groupes socioculturels du Sud-Ouest (68\% à $82 \%$ ) qu'au sein des groupes socioculturels du Sud-Est (17\% à $44 \%$ ). Cependant, le nombre moyen d'individus par propriétaire est moins élevé au sein des groupes socioculturels du Sud-Ouest qu'au sein des groupes socioculturels du Sud-Est. Il faut ajouter à ces constats que $S$. dulcificum n'est généralement pas récolté dans la nature, mais cultivé dans les champs et jardins de case. En conséquence, l'hypothèse des connaissances déterminées par l'abondance de la ressource est mise en défaut, et les tendances observées pourraient être expliquées par une tendance inverse, la connaissance des usages de la plante déterminant son abondance. En d'autres termes, les propriétaires au sein des groupes socioculturels du Sud-Est posséderaient plus d'individus de $S$. dulcificum parce qu'ils connaissent mieux les utilisations et la valeur de la plante et la cultivent donc davantage. Cette hypothèse pourrait être investiguée par l'étude des causes de variation des connaissances locales et de la valeur d'usage de S. dulcificum entre groupes socioculturels du Sud-Est et du Sud-Ouest du Bénin. Il faut aussi noter que ce gradient de connaissance et d'usage coïncide avec le gradient pluviométrique du Sud-Bénin (décroissant de 1300 mm/an à l'Est à 900 mm/an à l'Ouest) (Adomou et al., 2005). Mais cela n'indique pas pourquoi les connaissances suivent un tel gradient.

Un résultat singulier est la connaissance des utilisations et l'usage intense des parties de S. dulcificum par les Holli, dont aucun enquêté ne possédait un individu de la plante. Ceci corrobore en revanche les résultats d'autres études présentant ce groupe socioculturel comme détenteur particulier de savoir endogène au Bénin (Codjia et al., 2005 ; Lokonon, 2008). Ce statut particulier peut s'expliquer ici non seulement par la mobilité des Holi dans toute l'aire d'étude, mais aussi et surtout par leur cohabitation avec plusieurs autres groupes culturels (INSAE, 2003), ce qui faciliterait les échanges interculturels de connaissances (Avocèvou-Ayisso et al., 2011).

\section{La valeur d'usage de $S$. dulcificum varie au sein des groupes socioculturels}

Des variations intra-culturelles significatives de la valeur d'usage de $S$. dulcificum sont notées selon le sexe, l'âge et le domaine d'activité des enquêtés. L'étude s'inscrit dans le sillage de nombreux travaux qui ont observé que les caractéristiques individuelles comme l'âge, le sexe, les rôles et les responsabilités aux niveaux familial et communautaire, la profession, les aptitudes et les capacités intellectuelles, l'accès et le contrôle sur les ressources naturelles, peuvent influencer la connaissance de ces ressources et leur utilisation au sein d'une communauté (Lougbégnon et al., 2011).

\section{Les personnes âgées et adultes utilisent plus intensément les parties de $\boldsymbol{S}$. dulcificum}

Les connaissances sur $S$. dulcificum sont concentrées chez les personnes de 30 ans et plus, qui ont également plus intensément recours aux usages que les plus jeunes. Ce résultat soutient l'hypothèse de connaissances 
dépendantes de l'âge (Souto et Ticktin, 2012), mais pourrait également indiquer un risque d'érosion à long terme des savoirs endogènes portant sur cette espèce. Cependant, du fait que les connaissances s'accumulent dans le temps et se transmettent d'une génération à une autre (De Albuquerque et Hanazaki, 2009 ; Beltrán-Rodríguez et al., 2014), il est possible que les plus jeunes atteignent plus tard le même niveau de connaissance que les adultes et les vieux, si la chaîne de transmission des savoirs traditionnels ne se rompt pas. Une analyse transversale des connaissances et des usages dans le temps pourrait apporter plus de précision sur les risques de perte de connaissances traditionnelles sur $S$. dulcificum.

\section{Les praticiens de la médecine traditionnelle utilisent davantage $S$. dulcificum que les autres acteurs}

Les résultats des tests statistiques réalisés ont montré que les praticiens de la médecine traditionnelle utilisaient plus intensément les parties de $S$. dulcificum que les personnes actives dans d'autres domaines (VUE : DF $=3$; Chi-2 = 21,44; $P<0,001)$. Ces résultats s'accordent avec l'implication des praticiens de la médecine traditionnelle dans de nombreuses études ethnobotaniques, en raison de leur connaissance avérée des usages traditionnels des plantes et des recettes pour leur mise en œuvre (Semenya et al., 2012 ; Laleye et al., 2015).

\section{S. dulcificum est une ressource de subsistance}

En dépit du grand nombre d'utilisations potentielles répertoriées (64 usages), S. dulcificum reste sous-utilisé au Bénin, tout comme dans le reste de son aire de distribution en Afrique (Achigan-Dako et al., 2015). S. dulcificum n'est actuellement impliqué que dans des usages de subsistance, notamment la consommation du fruit comme édulcorant par les enfants, l'utilisation de la feuille et de la racine pour le traitement de troubles du système urogénital et d'infections.

Comme pour d'autres espèces fruitières indigènes tels le baobab (Assogbadjo, 2006) et le tamarinier (Fandohan et al., 2010), les femmes sont les acteurs les plus impliqués dans la commercialisation des parties de S. dulcificum. Le fruit est la seule partie de $S$. dulcificum ayant une valeur économique significative pour les populations locales du Sud-Bénin, avec un circuit de commercialisation relativement court et son caractère périssable (le fruit) comme contrainte majeure à sa vente. Sa commercialisation au cours d'une saison rapporte deux fois plus aux détaillants (16,97 USD) qu'aux semi-grossistes (8,70 USD). Considérant les deux fructifications annuelles de l'espèce, les acteurs impliqués dans le commerce des fruits peuvent en moyenne réaliser un revenu annuel inférieur à 30 USD. II apparaît ainsi que la commercialisation des parties de $S$. dulcificum ne constitue pas une importante activité génératrice de revenu pour les populations.

Cependant, avec l'intérêt économique grandissant que connaît la miraculine et d'autres composés extraits de $S$. dulcificum (Chen et al., 2010 ; Achigan-Dako et al., 2015 ; Shi et al., 2016), sa domestication et son intégration dans les politiques de production agricole, l'organisation du marché et la production de fruits pour les industries pharmaceutique, cosmétique et agroalimentaire, pourraient augmenter sa contribution au revenu des ménages ruraux et faire de $S$. dulcificum une espèce de rente de choix.

\section{Conclusion}

Synsepalum dulcificum est une espèce à usages multiples, participant à la couverture de besoins alimentaires et médicaux des populations du Sud-Bénin. En dépit de son potentiel sur les plans alimentaire, médicinal et cosmétique, la faible valeur économique de $S$. dulcificum ainsi que les difficultés liées à sa propagation (et plus généralement à sa sylviculture) constituent des contraintes à lever avant son intégration dans les systèmes agroforestiers locaux et le développement d'une chaîne de valeur par la mise en place d'une véritable filière (production, commercialisation, transformation et distribution).

Une telle perspective requiert également des investigations sur les variations morphologiques et génétiques de la plante pour guider la sélection des meilleurs cultivars conformément aux besoins des usagers de la plante. Par exemple, tenant compte de la distribution inégale de la valeur d'usage de la plante, des cultivars pourraient être développés pour la production de feuilles ou de racines destinées aux commerçants et praticiens de la médecine traditionnelle qui apparaissent comme les auteurs principaux de prélèvements destructeurs.

\section{Remerciements}

Ce travail a été réalisé sur fonds propres des auteurs et grâce à un soutien partiel du Conseil pour le développement de la recherche en sciences sociales en Afrique (CODESRIA) (NWG/01/2013).

\section{Références bibliographiques}

Achigan-Dako E. G., Tchokponhoué D. A., N'Danikou S., Gebauer J., Vodouhè R. S., 2015. Current knowledge and breeding perspectives for the miracle plant Synsepalum dulcificum (Schum. et Thonn.) Daniell. Genetic Resources and Crop Evolution, 62 (3): 465-476.

Adomou A. C., 2005. Vegetation patterns and environmental gradients in Benin: implications for biogeography and conservation. PhD thesis, Wageningen University, Wageningen, Netherlands.

Adomou A. C., Agbani O. P., Sinsin B., 2011. Plants. In: Neuenschwander P., Sinsin B., Goergen G. (éds). Protection de la Nature en Afrique de l'Ouest : Une Liste Rouge pour le Bénin / Nature Conservation in West Africa: Red List for Benin. Ibadan, Nigeria, International Institute of Tropical Agriculture, 21-46.

Akoègninou A., Van Der Burg W. J., Van Der Maesen L. J. G., Adjakidjè V., Essou J. P., Sinsin B., Yèdomonhan H., 2006. Flore analytique du Bénin. Cotonou et Wageningen, Backhuys Publishers, $1034 \mathrm{p}$.

Assogbadjo A. E., 2006. Importance socio-économique et étude de la variabilité écologique, morphologique, génétique et biochimique du baobab (Adansonia digitata L.) au Bénin. PhD thesis, Ghent University, Faculty of Bioscience Engineering, Belgium. 
Assogbadjo A. E., Glèlè Kakaï R., Chadare F. J., Thomson L., Kyndt T., Sinsin B., Van Damme P., 2008. Folk classification, perception and preferences of baobab products in West Africa: consequences for species conservation and improvement. Economic Botany, 62: 74-84.

Assogbadjo A. E., Kyndt T., Chadare F. J., Sinsin B., Gheysen G., Eyog Matig O., Van Damme P., 2009. Genetic fingerprinting using AFLP cannot distinguish traditionally classified baobab morphotypes. Agroforestry Systems, 75: 157-165.

Avocèvou-Ayisso C., Avohou T. H., Oumorou M., Dossou G., Sinsin B., 2011. Ethnobotany of Pentadesma butyracea in Benin: A quantitative approach. Ethnobotany Research and Applications, 10: 151-166.

Beltrán-Rodríguez L., Ortiz-Sánchez A., Mariano N. A., Maldonado-Almanza B., Reyes-García V., 2014. Factors affecting ethnobotanical knowledge in a mestizo community of the Sierra de Huautla Biosphere Reserve, Mexico. Journal of Ethnobiology and Ethnomedicine, 10: 14.

Bonou A., Adegbidi A., Sinsin B., 2013. Valeur économique des Produits Forestiers Non Ligneux (PFNL) au Bénin. Éditions universitaires européennes, $101 \mathrm{p}$.

Chen C. Y., Wang Y. D., Wang H. M., 2010. Chemical constituents from the leaves of Synsepalum dulcificum. Chemistry of Natural Compounds, 46 (3): 495.

Chen C.-C., Liu I.-M., Cheng J.-T., 2006. Improvement of insulin resistance by miracle fruit (Synsepalum dulcificum) in fructose-rich chow-fed rats. Phytotherapy Research, 20 (11): 987-992.

Codjia J. T. C., Vihotogbé R., Houéssou L. G., 2005. Analyse des déterminants socioéconomiques de l'état de conservation de l'espèce Irvingia gabonensis dans les systèmes agroforestiers traditionnels au Sud-Bénin. Institut national des recherches agricoles du Bénin (INRAB), Programme régional Sud-Centre du Bénin, Recherche agricole pour le développement, $35 \mathrm{p}$.

Dagnelie P., 1998. Statistiques théoriques et appliquées. Bruxelles, Belgique, De Boeck Services, 517 p.

De Albuquerque U. P., Hanazaki N., 2009. Five problems in current ethnobotanical research - and some suggestions for strengthening them. Human Ecology, 37 (5): 653-661.

De Albuquerque U. P., de Souza Araujo T. A., Ramos M. A., do Nascimento V. T., de Lucena R. F. P., Monteiro J. M., Alencar N. L., de Lima Araujo E., 2009. How ethnobotany can aid biodiversity conservation: Reflections on investigations in the semi-arid region of $\mathrm{NE}$ Brazil. Biodiversity and Conservation, 18 (1): 127-150.

De Mendiburu F., 2014. Agricolae: Statistical Procedures for Agricultural Research. R package version 1.2-0.

Espinosa M. M., Bieski I. G. C., de Oliveira Martins D. T., 2012. Probability sampling design in ethnobotanical surveys of medicinal plants. Revista Brasileira de Farmacognosia / Brazilian Journal of Pharmacognosy, 22 (6): 1362-1367.

Fandohan B. A., Assogbadjo A. E., Glele Kakaï R., Kyndt T., de Caluwé T. E., Codjia J. T. C., Sinsin B., 2010. Women's Traditional Knowledge, Use Value, and the Contribution of Tamarind (Tamarindus indica L.) to Rural Households' Cash Income in Benin. Economic Botany, 64 (3): 248-259.

Fandohan B., Assogbadjo A. E., Glele Kakaï R., Kyndt T., Sinsin B. 2011. Quantitative morphological descriptors confirm traditionally classified morphotypes of Tamarindus indica L. fruits. Genetics Resources and Crop Evolution, 58: 299-309.

Gouwakinnou G. N., Lykke A. M., Assogbadjo A. E., Sinsin B., 2011. Local knowledge, pattern and diversity of use of Sclerocarya birrea. Journal of Ethnobiology and Ethnomedicine, 7 (8): 1746-4269.

Honfo H., Tovissodé F. C., Gnanglè C., Mensah S., Salako V. K., Assogbadjo A. E. et al., 2015. Traditional Knowledge and Use Value of Bamboo in Southeastern Benin: Implications for sustainable management. Ethnobotany Research and Applications, 14: 139-153.

IARC, 1980. Some non-nutritive sweetening agents. Vol. 22. Lyon, France, WHO, $189 \mathrm{p}$
Inglett G. E., May J. F., 1968. Tropical plants with unusual taste properties. Economic Botany, 22: 326-331.

INSAE, 2003. Troisième recensement général de la population et de l'habitation (rgph-3). Résultats définitifs : caractéristiques générales de la population. Cotonou, Bénin, INSAE, $404 \mathrm{p}$.

Jaenicke H., Beniest J., 2002. Vegetative tree propagation in agroforestry. Training guidelines and references. Nairobi, Kenya, ICRAF, $130 \mathrm{p}$.

Joyner G., 2006. The Miracle Fruit. In: Scott P. (ed.). Quandong Magazine of the West Australian Nut and Tree Crop Association. Subiaco, West Australia, West Australian Nut and Tree Crop Association, 15.

Kalinganire A., Weber J. C., Uwamariya A., Kone B., 2008. Improving rural livelihoods through domestication of indigenous fruit trees in the parklands of the Sahel. In: Akinnifesi F. K., Leakey R. R. B., Ajayi O., Sileshi G., Tchoundjeu Z., Matakala P., Kwesiga F. (eds). Indigenous fruit trees in the tropics: Domestication, utilization and commercialization. Oxford, United Kingdom, CABI Publishing, 186-203.

Laleye F. O. A., Mensah S., Assogbadjo A. E., Ahissou H., 2015. Diversity, knowledge, and use of plants in traditional treatment of diabetes in the Republic of Benin. Ethnobotany Research and Applications, 14: 231-257.

Lokonon B. E., 2008. Structure et ethnobotanique de Dialium guineense Willd., Diospyros mespiliformis Hochst. ex A. Rich. et Mimusops andongensis Hiern. en populations dans le Noyau Central de la Forêt Classée de la Lama (Sud-Bénin). Thèse d'ingénieur agronome, FSA/UAC, Bénin, 89 p.

Lougbégnon T. O., Tente B. A. H., Amontcha M., Codjia J. T. C., 2011. Importance culturelle et valeur d'usage des ressources végétales de la réserve forestière marécageuse de la vallée de Sitatunga et zones connexes. Bulletin de la Recherche Agronomique du Bénin, 70: 35-46.

Masson L., 2014. Phenolic acids as natural antioxidants. In: Praksh D., Sharma G. (eds). Phytochemicals of neutraceutical importance. Wallingford, United Kingdom, CAB International, 196-207.

N'danikou S., Achigan-Dako E. G., Wong L. L. G., 2011. Eliciting local values of wild edible plants in Southern Benin to identify priority species for conservation. Economic Botany, 65 (4): 381-395.

Ogunsola K. E., Ilori C. O., 2008. In vitro propagation of miracle berry (Synsepalum dulcificum Daniel) through embryo and nodal cultures. African Journal of Biotechnology, 7 (3): 244-248.

Oumorou M., Dah Dovonon J., Aboh B. A., Hounsou Kaka M., Sinsin B., 2010. Contribution à la conservation de Synsepalum dulcificum : régénération et importance socioéconomique dans le département de l'Ouémé (Bénin). INRA, LEA, EPAC, 102 p.

Phillips O., Gentry A. H., 1993. The useful plants of Tambopata, Peru. II. Additional hypothesis testing in quantitative ethnobotany. Economy Botany, 47 (1): 33-43.

$R$ Core Team, 2014. A language and environment for statistical computing. Vienna, Austria, R Foundation for Statistical Computing.

Semenya S., Potgieter M., Tshisikhawe M., Shava S., Maroyi A., 2012. Medicinal utilization of exotic plants by Bapedi traditional healers to treat human ailments in Limpopo province, South Africa. Journal of Ethnopharmacology, 144 (3): 646-655.

Sharma G., Prakash D., Gupta C., 2014. Phytochemicals of neutraceutical importance: do they defend against diseases? In: Prakash D., Sharma G. (eds). Phytochemicals of nutraceutical importance. Wallingford, United Kingdom, CAB International, 1-19.

Shi Y.-C., Lin K.-S., Jhai Y.-F., Lee B.-H., Han Y., Cui Z., Hsu W.-H., Wu S.-C., 2016. Miracle Fruit (Synsepalum dulcificum) Exhibits as a Novel Anti-Hyperuricaemia Agent. Molecules, 21 (140): 1-11.

Souto T., Ticktin T., 2012. Understanding interrelationships among predictors (age, gender, and origin) of local ecological knowledge. Economic Botany, 66 (2): 149-164.

Venables W. N., Ripley B. D., 2002. Modern Applied Statistics with S. Fourth Edition. New York, USA, Springer. 